\title{
COMPORTAMIENTO DE LA QUÍMICA INORGÁNICA DE LOS CARBONES BITUMINOSOS EN EL SINCLINAL CHECUA - LENGUAZAQUE
}

\section{Behavior of the inorganic chemistry of bituminous coals in the Checua - Lenguazaque syncline}

\author{
David Alejandro Herrera Carvajal'1, John Elkin Rincón Bayona², Sandra Manosalva Sánchez ${ }^{3}$ \\ 1-2Universidad Pedagógica y Tecnológica de Colombia, Escuela de Ingeniería Geológica, Colombia. \\ ${ }^{3}$ Universidad Pedagógica y Tecnológica de Colombia, Escuela de Ingeniería Geológica, Directora del Grupo de \\ Investigación de Ingeniería Geológica INGEOLOG, Colombia.Email: ${ }^{1} d a v i d . h e r r e r a 01 @ u p t c . e d u . c o$, \\ 2john.rincon01@uptc.edu.co, ${ }^{3}$ sandra.manosalva@uptc.edu.co
}

(Recibido abril 15 de 2019 y aceptado mayo 01 de 2020)

\section{Resumen}

La Espectrometría de Fluorescencia de Rayos X, es una técnica que permite obtener los porcentajes de la química inorgánica presente en los carbones del flanco occidental del sinclinal Checua- Lenguazaque. Con base en los resultados obtenidos, es posible determinar el comportamiento de la química inorgánica de estos carbones, tanto a profundidad como en su extensión, permitiendo una mejor comprensión acerca de las variaciones químicas y conjuntamente determinar los carbones con mejores características de coquización en base a los factores Fouling y Slagging.

Palabras clave: espectrometría de fluorescencia de rayos $X$, formación uaduas, fouling y slagging, química inorgánica del carbón, sinclinal Checua - Lenguazaque.

\begin{abstract}
$X$ - Ray Fluorescence Spectrometry is a technique that allows to obtain the percentages of the inorganic chemistry present in the coals of the western flank of the Checua- Lenguazaque syncline. Based on the results obtained, it is possible to determine the behavior of the inorganic chemistry of these coals, both in depth and in their extension, allowing a better understanding of the chemical variations and jointly determine the coals with better coking characteristics based on Fouling and Slagging factors.
\end{abstract}

Key words: X-Ray fluorescence spectrometry, Guaduas formation, fouling and slagging, coal inorganic chemistry, ChecuaLenguazaque syncline.

\section{INTRODUCCIÓN}

El sinclinal Checua - Lenguazaque es una cuenca carbonífera que comparte frontera con los departamentos de Boyacá, al oriente y de Cundinamarca, al occidente. La actividad minera en esta zona gira entorno a la formación Guaduas, la cual posee dos miembros carboníferos importantes en los cuales se concentra la gran mayoría de la explotación en este sector.
A pesar que petrográficamente los carbones de este sector resultan ser de gran calidad para la industria del acero, la parte inerte de estos, ha resultado ser un factor poco tenido en cuenta. Esto considerando que, los residuos generados durante la combustión del carbón, a la larga, tiene una gran influencia en el costo de mantenimiento de los hornos usados en el proceso de coquización.

Los compuestos inorgánicos, es decir la materia mineral 
presente en los carbones, tiene distintos orígenes, bien sea sin-deposicional, enriquecimiento diagenético y/o tectónico, y al momento de ser expuesto a las altas temperaturas dentro de los hornos, pueden generar estructuras volátiles que generan encostramiento en los hornos.

La química inorgánica también permite la determinación del aporte marino o continental a los carbones, siendo que a la formación guaduas se le ha atribuido un ambiente de depositación transicional anudado a turberas bajas, distribuidas más cerca o más lejos de la paleo línea de costa.

El análisis estadístico realizado sobre los compuestos inorgánicos de los carbones, permite observar la variabilidad química y el comportamiento de los mantos identificados en el área de estudio.

\section{ESTADO DEL ARTE}

\subsection{Ubicación de la Zona de Estudio}

El Sinclinal Checua - Lenguazaque, (figura 1) se encuentra al NE de Bogotá y al NW de la Sabana en forma de corredor alargado con orientación SW-NE, de ancho variable, limitado por los flancos de una gran estructura de extensión regional, conocida con el nombre de Sinclinal de Checua de la cual se deriva el nombre, comprendida entre las poblaciones de Nemocón al Suroeste y Samacá al Noreste.

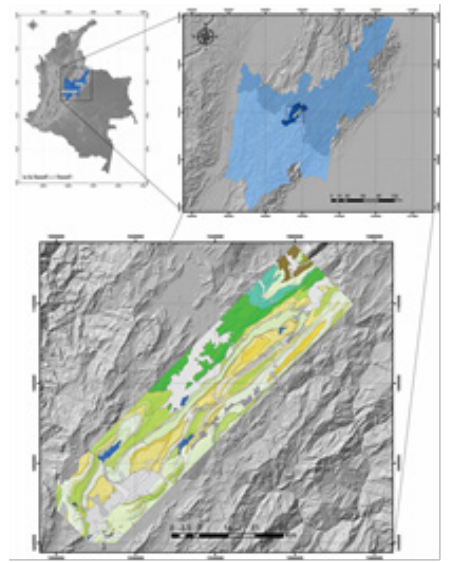

Figura 1. Ubicación general y específica del sinclinal Checua - Lenguazaque.

\subsection{Marco Geológico Local}

En el área de estudio afloran rocas sedimentarias, de edades que van del Cretácico al Paleógeno, representadas por las Formaciones: Conejo, Plaeners, Labor-Tierna, Guaduas, Cacho, Bogotá, Regadera y Cuaternario.

La unidad de estudio fue la Formación Guaduas, cuya edad calculada la ubica en el límite Cretácico - Paleógeno, hace aproximadamente 65 M.a [1]. En esta formación se levantaron 3 secciones estratigráficas, en los municipios de Samacá, Guachetá y Sutatausa.

El rumbo promedio de la estructura es N35E y su anchura varia de $3.5 \mathrm{~km}$ (Cucunubá) a $12 \mathrm{~km}$ (entre las poblaciones de Tausa y Nemocón).

\subsection{Marco Estratigráfico Local}

Para este estudio se tomó como base los levantamientos de tres secciones estratigráficas descritas por [2], donde se dividió la formación en tres miembros: miembro inferior (K2E1g1), miembro medio (K2E1g2), y miembro Superior (K2E1g3).

\subsection{Modelo Sedimentario de los Mantos de Carbón de la} Formación Guaduas

Según [1-2-3-4] los carbones de la Formación Guaduas representan faces típicas de ambientes transgresivos parálicos (telmáticos) a limnotelmáticos, detrás de barrera (GI y TPI) con influjo de aguas ricas en calcio que aceleran la descomposición de la materia orgánica y, por lo tanto, la humificación de los precursores de la vitrinita. Lugar en el que se desarrolla un sistema de pantanos de bosques húmedos y secos con intermitencia de moderadas a altas inundaciones y con dominio de plantas arborescentes y herbáceas bajo condiciones ligeramente óxicas a anóxicas.

\subsection{Química Inorgánica en Carbones}

En [5-6] se enfatiza que el contenido mineral del carbón es la fracción inorgánica no combustible, que se compone de minerales que son ya sea detrítico o autigenético en su origen (figura 2), y que se introducen en carbón en la primera o segunda fase de la carbonización. Una gran 
variedad de minerales se puede encontrar en el carbón, comúnmente estos están dominados por los minerales de cuarzo, carbonato, hierro y arcilla con un diverso conjunto de minerales accesorios que pueden ser de la roca o de una fuente local.

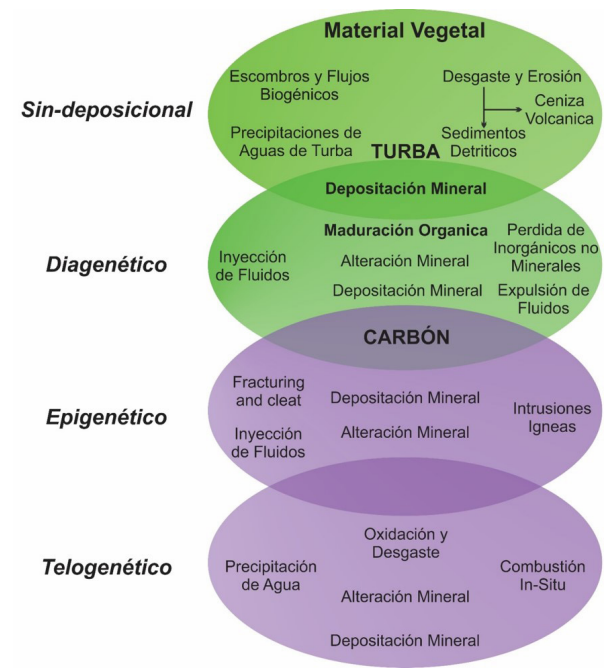

Figura 2. Clasificación del tipo y origen de la materia mineral en los carbones. [7].

\subsection{Fouling y Slagging}

El factor Fouling hace referencia a la formación de depósitos en superficies de calor de convección, tales como: sobrecalentadores y recalentadores.

Los factores Fouling y Slagging para los carbones se calculan siguiendo la metodología planteada en [8-9].

\subsubsection{Factor Slagging. Cuando se habla de Slagging} se refiere a la formación de depósitos fundidos o parcialmente fundidos en las paredes del horno o superficies de convección expuestas al calor radiante. Este se forma cuando las partículas de cenizas de fusión o ablandamiento no se enfrían a un estado sólido y cuando alcanzan la superficie de calor.

2.6.2 Factor Fouling. El factor Fouling hace referencia a la formación de depósitos en superficies de calor de convección, tales como sobrecalentadores y recalentadores. Es causado por la vaporización de elementos inorgánicos volátiles en el carbón durante la combustión. Cuando se absorbe el calor y las temperaturas disminuyen en el área de convección de la caldera, los compuestos formados por estos elementos se condensan en las partículas de cenizas y la superficie de calentamiento, formando un pegamento que inicia la deposición.

\subsection{Factores que Influencian la Generación de Fouling y Slagging}

Se pueden destacar tres factores principales: El tamaño del reducido del horno, la Fusibilidad de la Ceniza del Carbón en conjunto con la temperatura interna del horno y, por último, los elementos químicos presentes en las cenizas del carbón.

2.7.1 Elementos Presentes en la Ceniza del Carbón. La ceniza lignítica, que se define como que tiene más $\mathrm{CaO}+$ MgO que Fe2O3, es más fácil de depositar que la de las cenizas bituminosas, que se define como que tiene más Fe2O3 que la suma de $\mathrm{CaO}$ y $\mathrm{MgO}$.

Los metales alcalinos, sodio y potasio, durante mucho tiempo se han asociado con las tendencias de ensuciamiento (Slagging) de la ceniza de carbón. Las formas volátiles de estos elementos se vaporizan en el horno a la temperatura de combustión y reaccionan con el azufre en los gases de combustión y otros elementos en la ceniza forman compuestos que se depositan en las superficies de convección.

\section{METODOLOGÍA}

\subsection{Consolidación de la Información}

El proceso de consolidación, da inicio a la discriminación de las muestras de Carbón, escogiendo 41 muestras que serán objeto de estudio. Dentro de estas muestras seleccionadas para el análisis, se destacan un conjunto de 10 muestras piloto, las cuales ya disponían de análisis de química inorgánica, con la finalidad de servir como control de los datos obtenidos.

\subsection{Adecuación y preparación de muestras}

El proceso de acondicionamiento se realizó para las 41 
muestras seleccionadas y se dividió en tres fases.

La primera fase corresponde a la pulverización del Carbón hasta obtener una granulometría de 75 micras, la cual corresponde el tamaño de malla Taylor $n^{\circ} 200$.

La segunda fase corresponde a la incineración de las muestras previamente pulverizadas. Durante la incineración se busca obtener un concentrado de la materia inorgánica.

La tercera fase corresponde a la preparación del concentrado de materia inorgánica. Una vez se obtienen 9 gramos de ceniza del carbón se mezcla con 1 gramo de crema aglomerante LICOWAX, luego se prensan en un molde especial y se obtiene una pastilla cilíndrica, adecuada para encajar en los porta muestras del Espectrómetro EPSILON 4.

\subsection{Análisis de muestras}

Las muestras se disponen en grupos de 10 en el Espectrómetro EPSILON 4, en el cual procede a excitar los núcleos atómicos de los compuestos inorgánicos presentes en la pastilla, añadiéndoles energía, para lo cual se utilizan los rayos $x$ desprendidos de un cátodo de tungsteno.

Al ganar energía, los electrones experimentan el denominado salto cuántico, pasando de un orbital de menor energía (más cerca al núcleo atómico) a uno mayor energía (más lejos del núcleo atómico), e inmediatamente ocurre la perdida de la misma, con lo cual el electrón vuelve a un orbital de menor energía y por consiguiente se da el desprendimiento de un fotón y calor.

La longitud de onda del fotón es única dependiendo del tipo de elemento, y la lectura de estos por medio del detector nos permite determinar tanto el compuesto inorgánico como su cuantía porcentual dentro de la muestra. (Figura 3).

\subsection{Interpretación de la información}

Con estos datos se pueden discriminar los elementos mayores de los elementos menores, teniendo en cuenta la cantidad porcentual presente de estos elementos en las muestras analizadas. Se recalca que aquellos compuestos con cantidades representadas en partes por millón PPM no se tienen en cuenta para este proyecto dada su ínfima concentración en los carbones.

\begin{tabular}{|c|c|c|}
\hline Compound & Conc $L$ & Unit \\
\hline $\mathrm{Na} 2 \mathrm{O}$ & 0,238 & $\%$ \\
\hline $\mathrm{MgO}$ & 0,703 & $\%$ \\
\hline $\mathrm{Al} 2 \mathrm{O} 3$ & 18,813 & $\%$ \\
\hline $\mathrm{SiO} 2$ & 63,761 & $\%$ \\
\hline P2O5 & 0,378 & $\%$ \\
\hline $\mathrm{SO} 3$ & 0,592 & $\%$ \\
\hline d & $0,0 \mathrm{p}$ & $\mathrm{ppm}$ \\
\hline $\mathrm{K} 2 \mathrm{O}$ & 1,228 & $\%$ \\
\hline $\mathrm{CaO}$ & 1,187 & $\%$ \\
\hline $\mathrm{TOO} 2$ & 0,913 & $\%$ \\
\hline $\mathrm{Cr} 2 \mathrm{O} 3$ & $814,6 p$ & $\mathrm{ppm}$ \\
\hline $\mathrm{MnO}$ & $67,0 \mathrm{p}$ & $\mathrm{ppm}$ \\
\hline $\mathrm{Fe} 2 \mathrm{O} 3$ & 1,620 & $\%$ \\
\hline $\mathrm{NiO}$ & $257,5 \mathrm{p}$ & $\mathrm{ppm}$ \\
\hline CuO & $46,8 \mathrm{p}$ & $\mathrm{ppm}$ \\
\hline $\mathrm{ZnO}$ & $172,7 \mathrm{p}$ & $\mathrm{ppm}$ \\
\hline $\mathrm{Ga} 2 \mathrm{O} 3$ & $30,7 \mathrm{p}$ & $\mathrm{ppm}$ \\
\hline $\mathrm{GeO} 2$ & $14,5 \mathrm{p}$ & $\mathrm{ppm}$ \\
\hline As203 & $1,4 \mathrm{p}$ & $\mathrm{ppm}$ \\
\hline $\mathrm{MoO}_{3}$ & $40,5 \mathrm{P}$ & $\mathrm{ppm}$ \\
\hline $\mathrm{SnO} 2$ & $43,9 p$ & $\mathrm{ppm}$ \\
\hline $\mathrm{Eu} 2 \mathrm{O} 3$ & $109,6 p$ & $\mathrm{ppm}$ \\
\hline $\mathrm{OsO4}$ & $0,0 \mathrm{P}$ & $\mathrm{ppm}$ \\
\hline IrO2 & $0,0 \mathrm{p}$ & $\mathrm{ppm}$ \\
\hline $\mathrm{T} 12 \mathrm{O} 3$ & 0,9 & $\mathrm{ppm}$ \\
\hline $\mathrm{PbO}$ & $44,9 \mathrm{p}$ & $\mathrm{ppm}$ \\
\hline ThO2 & $23,9 p$ & $\mathrm{ppm}$ \\
\hline
\end{tabular}

\begin{tabular}{|c|c|c|}
\hline Compound & Conc & Unit \\
\hline $\mathrm{Na} 2 \mathrm{O}$ & 0,303 & $\%$ \\
\hline $\mathrm{MgO}$ & 0,104 & $\%$ \\
\hline $\mathrm{Al} 2 \mathrm{O} 3$ & 23,004 & $\%$ \\
\hline $\mathrm{SiO} 2$ & 64,418 & $\%$ \\
\hline P205 & 0,103 & $\%$ \\
\hline $\mathrm{SO} 3$ & 753,7 & ppm \\
\hline $\mathrm{Cl}$ & 41,8 & $\mathrm{ppm}$ \\
\hline K2O & 0,625 & $\%$ \\
\hline $\mathrm{CaO}$ & 0,189 & $\%$ \\
\hline $\mathrm{THO}_{2}$ & 1,047 & $\%$ \\
\hline $\mathrm{V} 205$ & 309,3 & $\mathrm{ppm}$ \\
\hline $\mathrm{Cr}_{2} \mathrm{O}$ & 470,2 & $\mathrm{ppm}$ \\
\hline $\mathrm{MnO}$ & 5,8 & $\mathrm{ppm}$ \\
\hline $\mathrm{Fe} 2 \mathrm{O3}$ & 1,878 & $\%$ \\
\hline NiO & 199,7 & $\mathrm{ppm}$ \\
\hline CuO & 58,0 & $\mathrm{ppm}$ \\
\hline $\mathrm{ZnO}$ & 82,5 & $\mathrm{ppm}$ \\
\hline $\mathrm{Ga} 2 \mathrm{O} 3$ & 40,0 & $\mathrm{ppm}$ \\
\hline $\mathrm{GeO2}$ & 51,9 & $\mathrm{ppm}$ \\
\hline As203 & 15,2 & $\mathrm{ppm}$ \\
\hline $\mathrm{Nb} 2 \mathrm{O} 5$ & 37,0 & $\mathrm{ppm}$ \\
\hline $\mathrm{MoO} 3$ & 32,3 & $\mathrm{ppm}$ \\
\hline $\mathrm{SnO} 2$ & 40,2 & $\mathrm{ppm}$ \\
\hline $\mathrm{Eu} 203$ & 79,5 & $\mathrm{ppm}$ \\
\hline $\mathrm{OsO} 4$ & 0,0 & $\mathrm{ppm}$ \\
\hline IrO2 & 0,0 & $\mathrm{ppm}$ \\
\hline $\mathrm{T} 12 \mathrm{O}$ & 1,3 & $\mathrm{ppm}$ \\
\hline $\mathrm{PbO}$ & 41,4 & $\mathrm{ppm}$ \\
\hline ThO2 & 26,4 & $\mathrm{ppm}$ \\
\hline
\end{tabular}

Figura 3. Datos obtenidos posterior al análisis por espectrometría de fluorescencia de rayos $\mathrm{x}$ realizado en las muestras de carbón. (A) Datos de química inorgánica de elementos mayores y menores obtenidos para la muestra $n^{\circ}$ 1998. (B) Datos de química inorgánica de elementos mayores y menores obtenidos para la muestra $n^{\circ} 2086$.

Para un mejor análisis estadístico y con la finalidad de evitar sesgo en la información, se introdujeron al análisis la información química referente a 45 muestras analizadas, previamente, por el Servicio Geológico Colombiano.

El análisis se llevó a cabo usando el programa IBM SPSS STADISTICS, en el cual se realizaron pruebas correlacionando bivariadas y observando su capacidad de correlación por el método de Spearman, explicado en [10-11].

Una vez encontradas las diferentes correlaciones se procede a realizar un gráfico de dispersión de puntos, que muestre la tendencia de estos y su relación bien 
sea directa o inversamente proporcional, asociando los datos de química Inorgánica presente en los carbones, correlacionándolos, principalmente, entre ellos y con algunas características físicas de los carbones, sobre todo con los factores Fouling y Slagging.

\subsection{Generación de mapas}

Los mapas fueron generados ubicando geográficamente los puntos donde las muestras habían sido recolectadas, asignado el valor promedio del compuesto químico para ese punto e interpolando los valores para toda el área de estudio. Estos mapas están distribuidos mostrando las tres secciones principales en las que se dividió el área de estudio, con la finalidad de mostrar la distribución de la química inorgánica en área del sinclinal Checua - Lenguazaque y el control de los ambientes de depositación, teniendo en cuenta que hacia el eje del Sinclinal los elementos depositados tienen un dominio, en su mayoría, continental y, en la parte distal del eje del sinclinal, los elementos depositados están controlados por un régimen mayormente marino.

\subsection{Distribución química por mantos de carbón}

Con el fin de obtener la distribución a profundidad de la química inorgánica de los mantos de carbón muestreados y analizados, se procedió a graficar los datos tratados en barras laterales, discriminándolos en elementos mayores y menores. (figura 4).

Con las barras laterales se modifican las columnas estratigráficas del sector de estudio. De esta forma, se puede hacer la lectura de la distribución a profundidad de la química inorgánica de los mantos de carbón.

Se debe tener en cuenta que, la sección Samacá no se tuvo en cuenta en este paso del proyecto, dado que los mantos muestreados en esta este sector presentan un alto grado de enriquecimiento mineral, siendo datos anómalos, que dificultan el análisis y, por lo tanto, fueron descartados.

Por los factores antes expuestos, se incluye la sección Cucunuba (correlacionable con la sección Sutatausa) y la sección Lenguazaque ubicada entre la sección Guachetá y la sección Sutatausa.
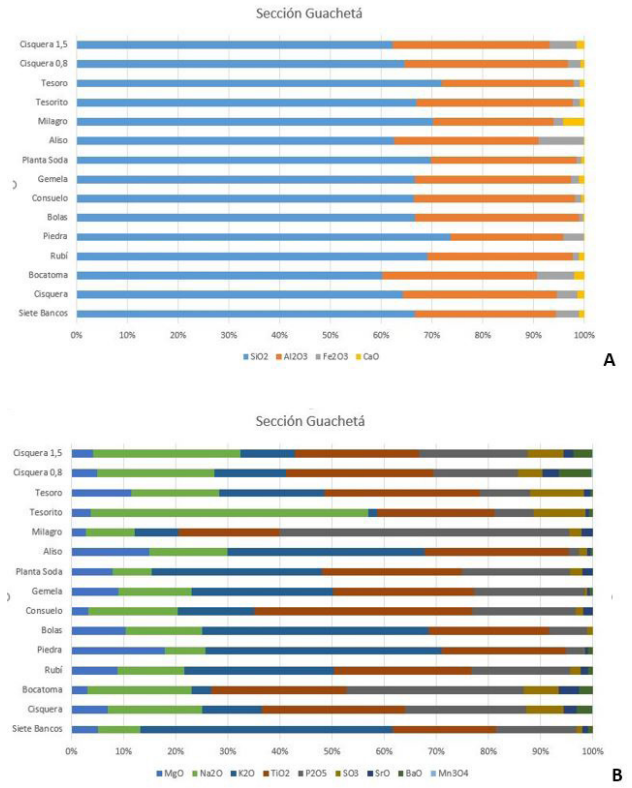

Figura 4. Barras de distribución de química inorgánica en los mantos de carbón de la sección Guachetá. (A) Distribución de elementos mayores. (B) Distribución de elementos menores.

\section{RESULTADOS}

\subsection{Gráficos de Correlación entre datos}

Las gráficas presentadas a continuación, exponen las correlaciones más importantes observadas: Oxido de Calcio vs Anhidrido Fosfórico (figura 5), Factor Fouling vs Óxido Férrico (figura 6), Factor Fouling vs Óxido Sódico (figura 7), Factor Fouling vs Óxido de Sílice (figura 8) y Factor Slagging vs Óxido Férrico (figura 9). 


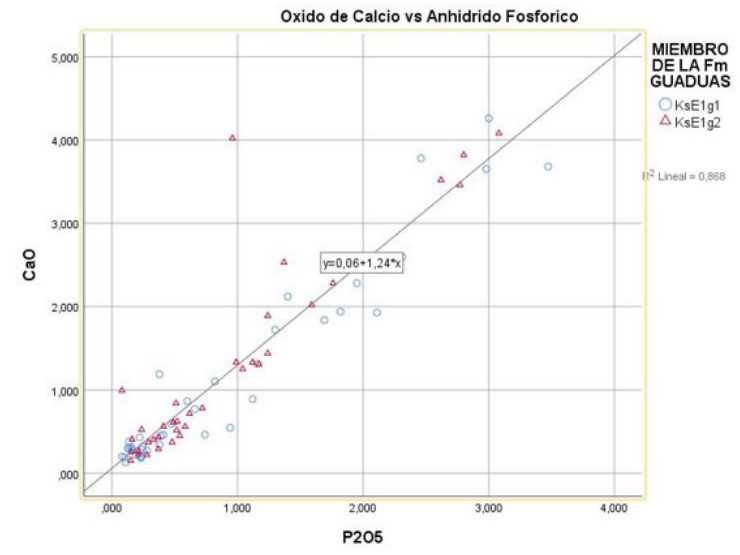

Figura 5. Gráfica de correlación directa entre el óxido de Calcio y el Anhídrido Fosfórico discriminando por miembro estratigráfico.

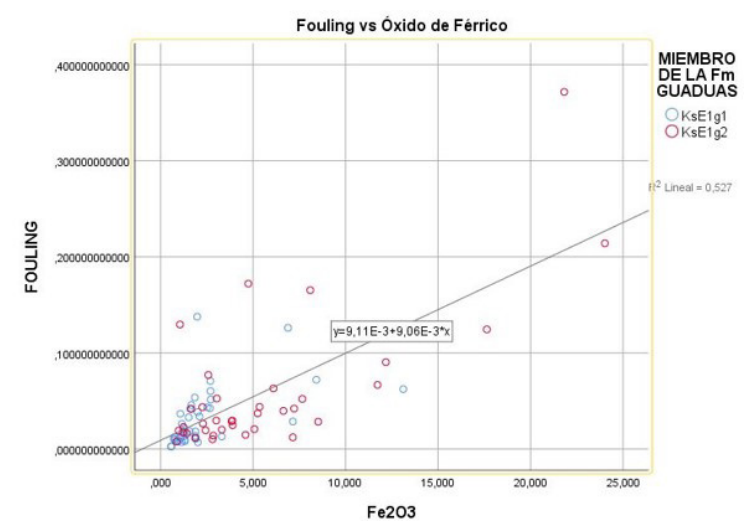

Figura 6. Gráfica de correlación directa entre el Factor Fouling y el Óxido Férrico discriminando por miembro estratigráfico.

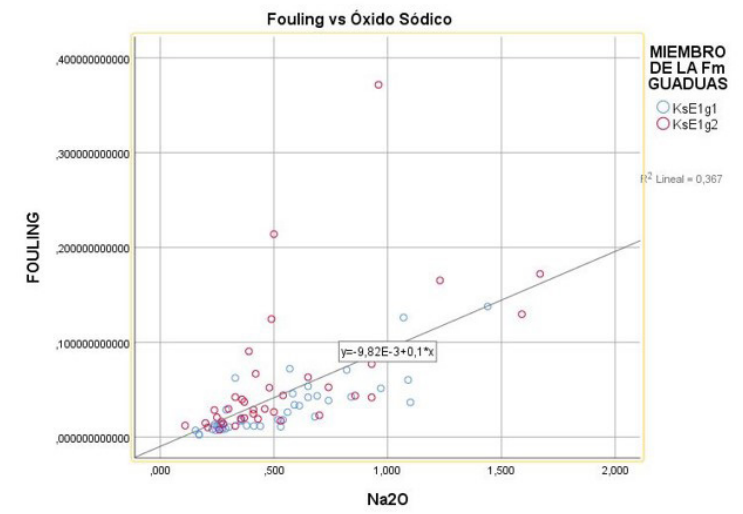

Figura 7. Gráfica de correlación directa entre el Factor Fouling y el Óxido Sódico discriminando por miembro estratigráfico.

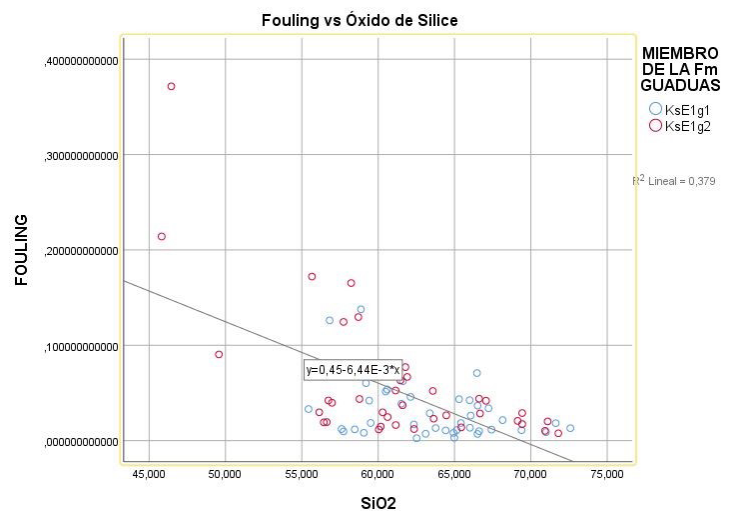

Figura 8. Gráfica de correlación inversa entre el Factor Fouling y el Óxido de Sílice discriminando por miembro estratigráfico.

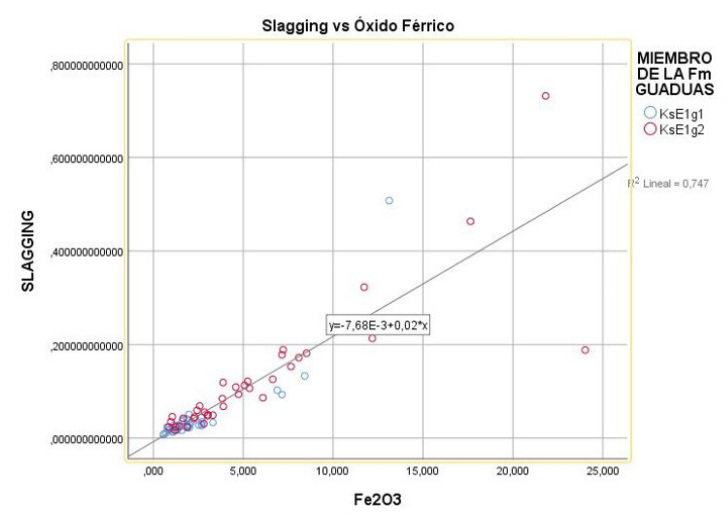

Figura 9. Gráfica de correlación directa entre el Factor Slagging y el Óxido Férrico discriminando por miembro estratigráfico.

\subsection{Resultados de la Sección Guachetá}

Los datos muestran que los elementos mayores (figura 10) son Silicio (60\% a $74 \%$ ), Aluminio (30-35 \%), Hierro (2 $-7 \%$ ) y Calcio ( $\leq 2 \%-3 \%)$. Elementos menores (figura 11 ) o iguales a 1\%, están en orden de abundancia, Potasio, Titanio, Sodio, Fósforo, Magnesio, Bario y Manganeso. 


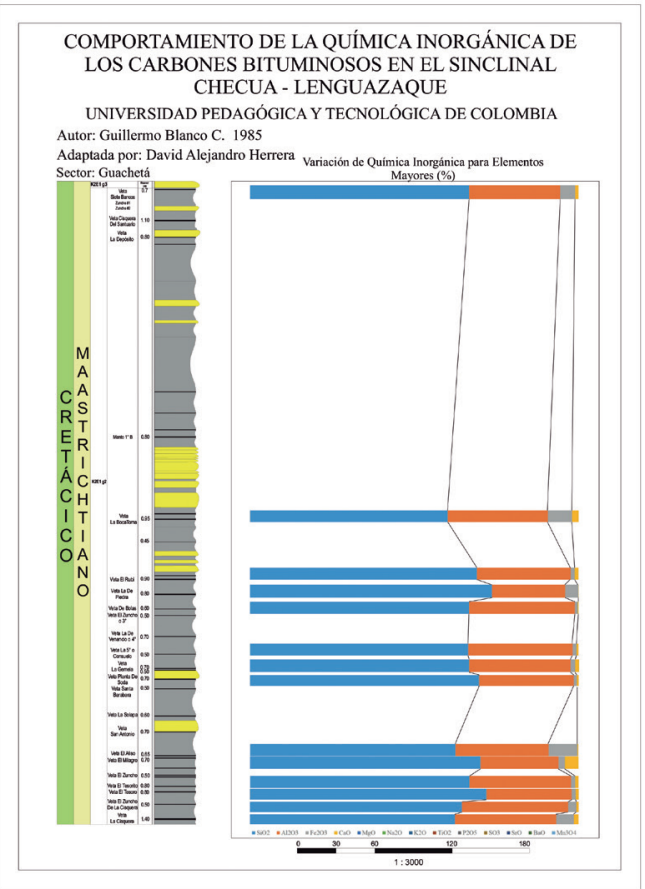

Figura 10. Columna Estratigráfica de la Sección Guachetá con distribución de elementos mayores.

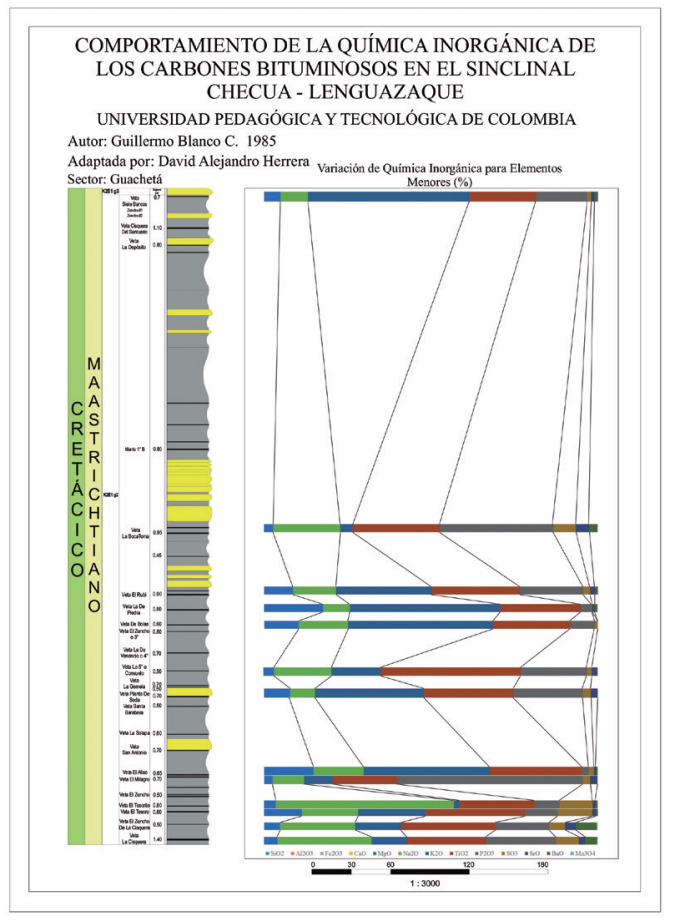

Figura 11. Columna Estratigráfica de la Sección Guachetá con distribución de elementos menores.

\subsection{Resultados de la Sección Lenguazaque}

Los datos muestran que los elementos mayores son Silicio (58 a $62 \%$ ), Aluminio (25-30\%), Hierro (2-9 \%) y Calcio $(\leq 2 \%)$. Elementos menores o iguales a $1 \%$, están en orden de abundancia, Potasio, Titanio, Fósforo, Sodio y Magnesio.

Al observar la distribución de elementos mayores en esta sección, no se encontró la existencia de una secuencia o comportamiento que siga la química, no obstante, al analizar el comportamiento de los elementos menores, es posible observar un aumento en el Magnesio, Sodio, Titanio, SO3, Estroncio, Bario y un descenso en el Potasio y el Fósforo.

\subsection{Resultados de la Sección Cucunuba - Sutatausa}

Los datos muestran que los elementos mayores son Silicio (61\% a $73 \%)$, Aluminio (25-35\%), Hierro (2-10 \%) y Calcio ( $\leq 2 \%$ - 3\%). Elementos menores o iguales a 1\%, están en orden de abundancia, Potasio, Titanio, Fósforo, Magnesio, Sodio, Bario y Manganeso.

En consecuencia, al observar la distribución de elementos tanto mayores como menores en esta sección, no se encontró la existencia de una secuencia o comportamiento de aumento o descenso que sigan los compuestos químicos. Estos aumentos y descensos irregulares de la concentración de estos compuestos permiten discernir que su fluctuación depende de factores diagenéticos y no sin-deposicionales.

\subsection{Mapas de Variación Química}

4.5.1 Óxido Férrico. Hacia el sector de Guachetá y Sutatausa se observa que las concentraciones más grandes de este compuesto están hacia el eje del sinclinal lo cual nos indica un aporte principalmente continental. Sin embargo, hacia el sector de Samacá esta distribución se pierde y se logran apreciar valores realmente altos, que nos indica una gran concentración de este compuesto en toda esta sección, posiblemente de origen epigenético o telogenético. (Figura 12). 


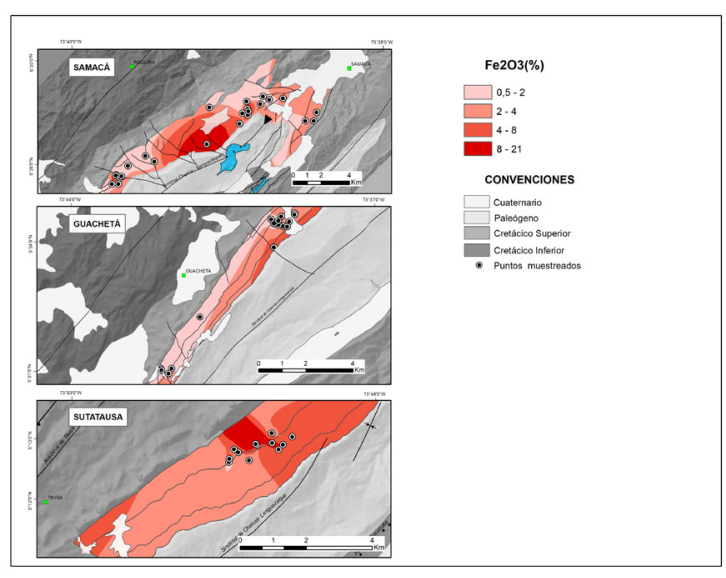

Figura 12. Mapa de distribución del Óxido Férrico para las tres secciones estratigráficas.

4.5.2. Óxido de Aluminio. Tanto en el sector de Samacá como se Sutatausa se puede observar que las mayores concentraciones están hacia el lado opuesto del eje del sinclinal, lo cual indica un aporte esencialmente marino. Sin embargo, en el sector de Guachetá esta distribución cambia radicalmente mostrando las mayores concentraciones hacia el eje del sinclinal, lo cual podría indicar un aporte importante de minerales arcillosos provenientes del continente. (Figura 13).

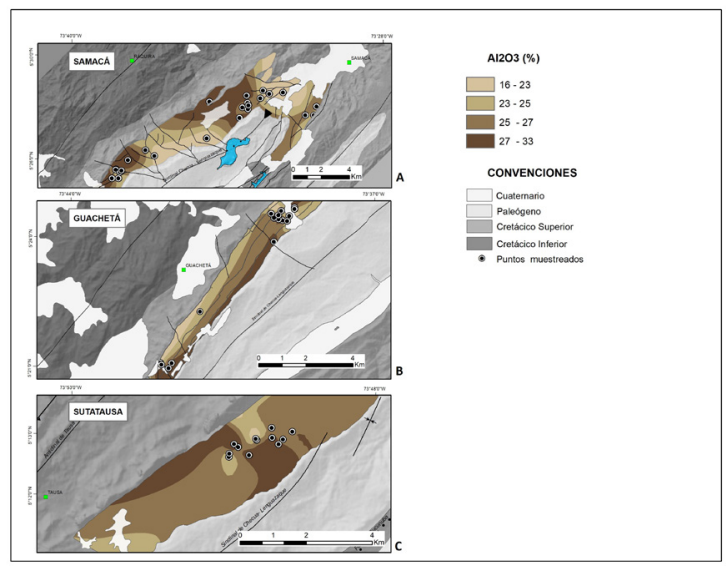

Figura 13. Mapa de distribución del Óxido de Aluminio para las tres secciones estratigráficas.

4.5.3. Óxido de Silicio. Hacia el sector de Samacá se observa que las mayores concentraciones están hacia el eje del sinclinal, lo cual indica que en este sector la depositación de este compuesto fue dominada por la parte continental. En el Sector de Guachetá las mayores concentraciones están en el lado opuesto al sinclinal, lo cual ilustra que el aporte a la cuenca en este sector fue dominado por un régimen marino. (Figura 14).

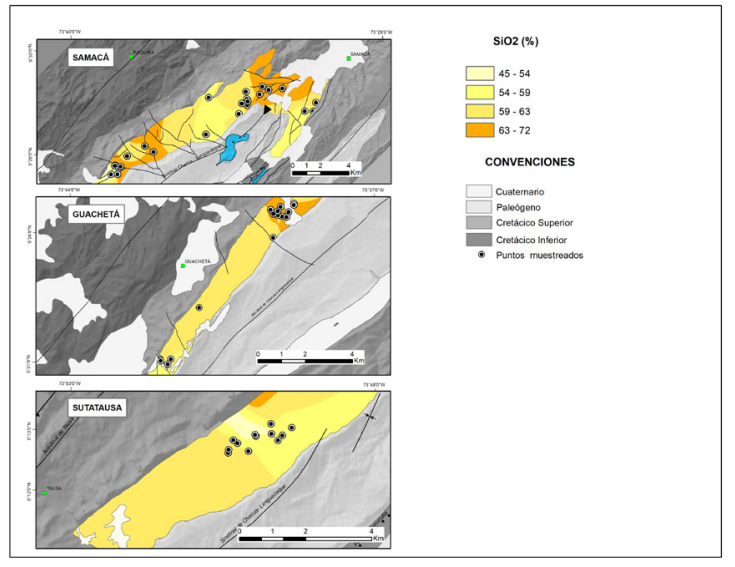

Figura 14. Mapa de distribución del Óxido de Sílice para las tres secciones estratigráficas.

4.5.4. Óxido de Calcio. Los mayores valores están en el lado opuesto del eje del sinclinal, indicando que su depositación a lo largo de toda la cuenca fue principalmente dominada por un régimen mareal, tal cual se espera que sea. (Figura 15).

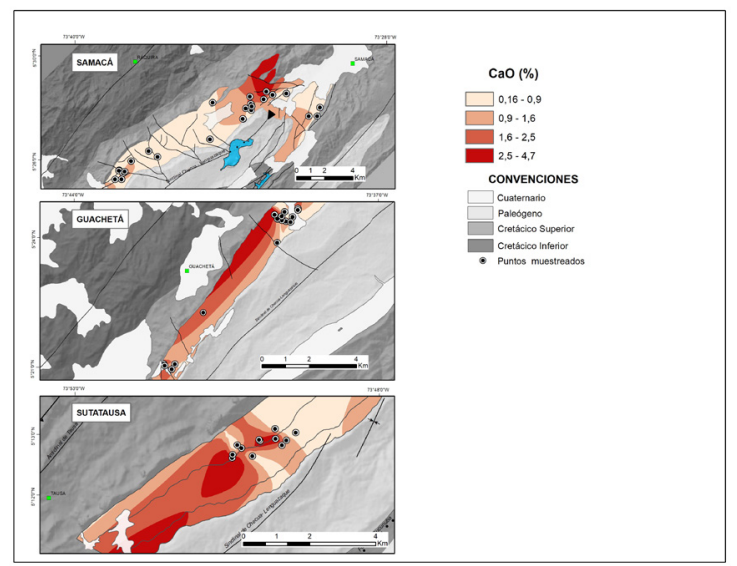

Figura 15. Mapa de distribución del Monóxido de Calcio para las tres secciones estratigráficas. 


\section{ANÁLISIS Y DISCUSIÓN DE RESULTADOS}

1. Los datos arrojados por el espectrómetro resultan ser concordantes con lo obtenido en la búsqueda bibliográfica, cuando se realizó la comparación mediante mantos y secciones, este proceso permitió encontrar datos anómalos (muy elevados o disminuidos), los cuales se aislaron para su posterior búsqueda en los mapas y determinar el origen de esta anomalía.

2. Las correlaciones permiten determinar cómo ciertos elementos tienen una influencia directa sobre el poder calorífico del carbón, es decir, elementos como el Na2O incrementan la capacidad de combustión del carbón, caso contrario el $\mathrm{K} 2 \mathrm{O}$ y el $\mathrm{MgO}$, los cuales entre mayor presencia tengan dentro del carbón menor, será la capacidad de combustión de este.

3. Elementos como el $\mathrm{Na} 2 \mathrm{O}$ y el Fe2O3 al ser sometidos a altas temperaturas generan volátiles semifundidos, que al hacer contacto con una superficie un poco más fría se solidifican, creando un encostramiento y ensuciamiento en las superficies expuestas a este tipo de semifundidos generados. Estos factores denominados Fouling y Slagging, resultan ser muy importantes en la industria siderúrgica, dado que de esto depende el tiempo de mantenimiento de los hornos de producción de coque, lo cual se puede traducir en pérdidas para la empresa.

4. Algunos compuestos químicos presentan una fuerte correlación entre ellos, debido a que el ambiente de depositación de estos suele ser muy similar. Este es el caso del $\mathrm{CaO}$ junto con el P2O5, para estos dos compuestos químicos se les ha atribuido un origen similar en el ámbito Geoquímico, hecho que sustenta la alta presencia de uno de ellos e indica directamente una fuerte presencia del otro.

5. En la sección estratigráfica Samacá - Ráquira, es posible ver anomalías químicas interesantes en el mapa que no parece seguir una distribución regular, esto puede deberse al hecho que esta sección en particular dentro del sinclinal, sufrió un fuerte fracturamiento y diaclasamiento debido al tectonismo intenso presente en esta zona.

6. Este intenso tectonismo se puede evidenciar también con el alto grado de maduración que presentan los carbones en esta sección estratigráfica. El diaclasamiento de los mantos de carbón facilita la entrada de agua escorrentía oxidando los mantos y el fallamiento, debido al calor y fricción generado la recristalización de familias minerales dentro de las cuales están implícitos los compuestos químicos acá analizados.

7. Las columnas estratigráficas nos permiten observar que no existe correlación alguna entre la química inorgánica y la profundidad a la que se encuentra el manto, asunto que permite deducir que estos compuestos químicos están principalmente regidos por las condiciones de depositación y la posterior diagénesis sufrida.

8. En la distribución de elementos mayores para la sección Guachetá (figura 11) se observa una tendencia entre el comportamiento del Sílice y del Aluminio, a medida que uno aumentó, el otro también; al igual que con el descenso, esta tendencia se pierde para con los demás elementos mayores, los cuales no muestran correlación respecto a estos dos primeros. Con los elementos menores (figura 12) se aprecia un aumento significativo del sodio en ciertos mantos a medida que se profundiza, lo que puede indicar un control de este elemento por las condiciones de depositación o cambios diagenéticos relacionados con la profundidad, ya sea la recristalización de minerales por acción del gradiente Geotermal o por acción de aguas subterráneas. A profundidad se aprecia también el aumento del SO3, Estroncio y Bario.

9. Al analizar los mapas generados es posible encontrar en algunas secciones estratigráficas, una distribución gradada de la concentración de un elemento químico en específico, este hallazgo permite identificar si el aporte de este elemento es principalmente continental o marino, teniendo siempre en cuenta la Paleo Línea costera. 


\section{CONCLUSIONES}

El método de fluorescencia de Rayos-X resulta muy efectivo a la hora de realizar análisis químico a los carbones, dado que la respuesta de cada compuesto al ser irradiado ya ha sido previamente estudiada y solo resta comparar los datos obtenidos.

La correlación directa que existe entre compuestos como Fe2O3, SiO2 y Na2O con el factor Fouling dan un excelente componente a la hora de determinar aquellos mantos de carbón que, al momento de ser llevado al horno, podrían generar un encostramiento en este, lo cual causa pérdidas importantes a las empresas por el costo del mantenimiento.

El factor Slagging resulta tener una correlación directa con el Fe203, por esta razón, y a causa del hecho que para calcular este factor se usa el Óxido Férrico, dado su alto contenido en la muestra, este suele tener una gran influencia en el cálculo del mismo.

El poder calorífico es uno de los factores que mayor correlación presenta con los compuestos químicos, tanto de forma directa como inversa. El MgO, K2O y las cenizas, reducen el poder calorífico de un carbón esto debido a que al ser incinerados estos compuestos resultan ser inertes. Por el contrario, el Na2O resulta ser un compuesto químico que posee la propiedad inherente de incrementar sustancialmente la combustión de otras sustancias, en este caso del carbón.

En la búsqueda de encontrar una explicación más certera para los valores anómalos hallados, se realizó un rastreo de dichos puntos dentro de los mapas elaborados. Durante dicho reconocimiento se encontró que varios de estos se ubicaban cerca o sobre fallas locales que afectan el sinclinal. Este enriquecimiento en ciertos minerales puede deberse directamente a la interacción de la falla con el manto en ese punto o a la infiltración de agua escorrentía que, al interactuar con el manto de carbón, lo oxida y enriquece en ciertos elementos químicos.
Las mayores concentraciones de la distribución de los óxidos de Hierro, Potasio, Magnesio y Sílice se encuentran hacia el centro del sinclinal, lo cual indica que su depositación dentro de la cuenca estaba controlada principalmente por un régimen continental, esto claro, al corroborar la influencia tanto continental, como mareal a la que estaba expuesta la cuenca.

Por último, cabe resaltar que, según lo observado durante la realización de este proyecto, la química inorgánica en los carbones esta principalmente ligada al ambiente de depositación de las turberas y las diagénesis posteriores, que a cualquier otro factor.

\section{REFERENCIAS}

[1] J. E. Mariño Martinez and E. Amaya, "Lithofacies cyclicity determination in the guaduas formation (Colombia) using Markov chains", Earth sci. res. j., vol. 20, no. 3, pp. B1-B9, Julio. 2016. https://doi. org/10.15446/esrj.v20n3.44429

[2] J. A. Barrera Pongutá, "Determinación de la madurez térmica de los carbones de la formación guaduas en el Sinclinal Checua - Lenguazaque". (Trabajo de pregrado). Universidad Pedagógica y Tecnológica de Colombia, Sogamoso. (2016). Disponible en: http:// repositorio.uptc.edu.co/handle/001/1691

[3] G. A. Sarmiento Pérez y C. L. Guatame, "Interpretación del Ambiente Sedimentario de los Carbones de la Formación Guaduas en el Sinclinal Checua-Lenguazaque a partir del análisis petrográfico", Geología Colombiana, pp. 41-58, enero. 2004

[4] J. S. Gómez Neita, M. D. López Carrasquilla, "Paleoenvironments of coals using organic petrography and their relationship with physicochemical properties, Guaduas formation, Checua-Lenguazaque syncline". (Trabajo de grado). Universidad Pedagógica y Tecnológica de Colombia, Sogamoso. (2017). Disponible en: http://repositorio. uptc.edu.co/handle/001/1875

[5] C. R. Ward, "Analysis, origin and significance of mineral matter in coal: An updated review". 
International Journal of Coal Geology, no. 165, p.p. 1-27. 2016. https://doi.org/10.1016/j. coal.2016.07.014

[6] L. Thomas, "Coal Sampling and Analysis". Coal Geology, L. Thomas, Ed. Reino Unido: John Wiley \& Sons, Ltd, 2012. pp. 137-149. DOI:10.1002/9781118385685

[7] M. C. Valentinuzzi, "Análisis por Fluorescencia de Rayos X: Implementación de Guías de Haces en Reflexión Total". Trabajo de Grado de Doctorado. Facultad de Matemática, Astronomía y Física. Universidad Nacional de Córdoba. Argentina. 2008.

[8] F. Frandsen, "Empirical Prediction of Ash Deposition Propensities in Coal-Fired Utilities". Department of Chemical Engineering Technical University of Denmark. Dinamarca, ene, 1997, pp. 3 - 6. Disponible en: https://www.osti.gov/etdeweb/ servlets/purl/594959

[9] Mohammad Zahari Sukimi Mat Zaid, Mazlan Abdul Wahid, Musa Mailah, Mohammad Amri Mazlan, and Aminuddin Saat, "Coal combustion analysis tool in coal fired power plant for slagging and fouling guidelines", AIP Conference Proceedings 2062, pp. 17. enero, 2019. https://doi.org/10.1063/1.5086575

[10] A. Badii, O. P. Guillen, S. Lugo, \& J. J. Aguilar Garnica, "Correlación No-Paramétrica y su Aplicación en la Investigaciones Científicas. Non-Parametric Correlation and Its Application in Scientific Research". International Journal of Good Conscience, vol. 9, no. 2, pp. 31-40, Agosto, 2014.

[11] M. G. Kendall, B. B. Simith, "Randomness and Random sampling numbers", pp. 103, 147-166, 1938. https://doi.org/10.2307/2980655 